\section{Lead Infective Endocarditis Caused by Acinetobacter baumannii}

\author{
Keywords: Acinetobacter, Coccobacilli, Oropharynx
}

Received: September 10, 2015, Accepted: September 18, 2015, Published: September 22, 2015

\section{Article}

The Acinetobacter species are ubiquitous gram-negative, coccobacilli that reside as a normal flora of the skin and oropharynx and can survive on dry inanimate objects for months. They are generally considered as opportunistic pathogens and often cause a variety of nosocomial infections [1]. We report a case of endocarditis in a patient who underwent pulse generator implantation for her permanent pacemaker caused by Acinetobacter baumannii complex.

A 40 years old female had a permanent pacemaker insertion done in 2004 for her congenital complete heart block. In 2014, she underwent pulse generator implantation following which she presented with complaints of fever and chest pain. There were no clinical features of embolic complications. Systemic examination and ECG were normal and repeated blood cultures were negative. Trans-esophageal echography revealed vegetations on Right Ventricular (RV) portion of pacemaker lead. Final diagnosis of Infective Endocarditis of RV pacemaker Lead was made (satisfying Duke Criteria). Vegetectomy with lead extraction was done and vegetations were received for culture and antibiotic sensitivity testing.

Samples were inoculated in three bottles of Brain Heart infusion broth with $0.04 \%$ sodium polyanethol sulphonate (HIMEDIA Laboratories, Mumbai). Within 24 hrs of inoculation at 370C, turbidity was noticed in all three bottles. Gram-stained smears showed gram-negative pleomorphic coccobacilli, which had non lactose fermenting colonies on MacConkey agar and nonhemolytic smooth colonies on blood agar. The isolate was non motile, catalase positive, and oxidase negative. It was identified as Acinetobacter baumannii complex based on oxidation of glucose, growth at $440 \mathrm{C}$ and resistance to penicillin and chloramphenicol [2].Antimicrobial sensitivity testing was performed as per Clinical and Laboratory Standard Institute recommendations by the Kirby-Bauer disc diffusion method [3]. The isolate was found sensitive to Co-trimoxazole, Ciprofloxacin, Levofloxacin, Amikacin and Imipenem. Patient was treated with Imipenem(500 mg IV 6hourly) and Amikacin(375mg IV 12 hourly), became asymptomatic and recovery was uneventful.
Maj (Dr) Gurpreet Singh Bhalla ${ }^{1}$, Col Naveen Grover ${ }^{2}$ and Brig $\mathrm{A} \mathrm{Garg}^{3}$

1 Dept of Microbiology, AFMC, Pune4110402, India

2 Dept of Microbiology, AFMC, Pune411040, India

3 Dept of Cardio Thoracic \& Vascular Surgery, MH (CTC), Pune-411040, India

Corresponding author: Maj (Dr) Gurpreet Singh Bhalla

Resident, Dept of Microbiology, AFMC, Pune-411040, India.

gs.indian5332@gmail.com

Tel: 8412822489

Citation: Bhalla MGS, Col Naveen Grover CN, Garg BA. Lead Infective Endocarditis Caused by Acinetobacter baumannii. J Infec Dis Treat. 2015, 1:1.

Health care associated Infective Endocarditis (IE) has emerged secondary to the introduction of new therapeutic modalities [4] like permanent pacemakers which are commonly used in cardiac rhythm management devices. Turbulence is an important risk factor and implantable intracardiac prosthetic material contributes to the same. In the past, sternotomy or thoracotomy was required to insert these devices. However, they are now implanted percutaneously using transvenous leads. Microbial contamination of device at the time of implantation is the most likely mechanism to explain infection. Once a pocket is colonized, bacteria migrate along the device to cause tunnel infection, bacteraemia or vegetations. Microorganisms adhere to the device and form biofilms which impair the host's immune response and poor penetration of antibiotics makes device removal mandatory to treat infection. The ability to form biofilms and acquire antibiotic resistance determinants makes infections with Acinetobacter a significant problem. Colonization with Acinetobacter can lead to deeper and serious infections when the host's barriers are breached. Most likely in our patient, colonization led to pocket site infection followed by spread and RV lead to IE. With the exception of Staphylococcus aureus, 
bacteraemia is relatively rare from infected pacemakers; [5] hence culture from the lesion becomes mandatory to identify the offending pathogen and its antimicrobial susceptibility. As hospital environment is an important reservoir of nosocomial infections, Acinetobacter infections demonstrate the importance of nosocomial infection control interventions. 


\section{References}

1 Towner KJ (1997) Clinical importance and antibiotic resistance of Acinetobacter spp. J Med Microbiol 46: 721-46.

2 Lahiri KK, Mani NS, Purai SS (2004) Acinetobacter spp as nosocomial pathogen: Clinical significance and antimicrobial sensitivity MJAFI 60: 7-10.

3 Performance Standards for antimicrobial susceptibility testing.
Clinical and Laboratory Standards Institute (CLSI) M100-S23 2013; 33: 66-7.

4 Von Reyn CF, Levy BS, Arbeit RD, Friedland G, Crumpacker CS (1981) Infective endocarditis: an analysis based on strict case definitions. Ann Intern Med 94: 505-18.

5 Chamis AL, Peterson GE, Cabell CH, Corey GR, Sorrentino RA, et al. (2001) Staphylococcus aureus bacteremia in patients with permanent pacemakers or implantable cardioverter-defibrillators. Circulation 104: 1029-1033. 